Operation bei Schlaganfall

\section{Hirnblutung - offen oder endoskopisch entfernen?}

\author{
Der Zustand verschlechtert sich, das Hämatom muss aus dem Hirn, \\ aber wie? Bei oberflächennahen Blutungen hat nach wie vor eine \\ minimalinvasive Kraniotomie Vorteile, bei tieferen Blutungen sind \\ moderne endoskopische Verfahren eine Option.
}

W as bringt eine Operation bei Patienten mit intrazerebralen Blutungen (ICB)? In der vor etwa acht Jahren veröffentlichten Studie STICH („International Surgical Trial in Intracerebral Haemorrhage") spielte es keine Rolle, ob die über 1.000 Patienten mit spontan aufgetretener supratentorieller ICB entweder innerhalb von 24 Stunden operiert oder konservativ behandelt wurden: Sechs Monate nach dem Blutungsereignis war kein Unterschied zwischen den beiden Behandlungsgruppen festzustellen, nur etwa $25 \%$ der Patienten erreichten ein günstiges funktionelles Ergebnis, etwa $37 \%$ waren gestorben. Allerdings gab es auch Kritik an der Studie: Teilnehmen durften nämlich nur solche Patienten, bei denen keine eindeutige Indikation zur Hämatomausräumenden Operation bestand und sich die behandelnden Ärzte über das beste Vorgehen unsicher waren. Schaut man sich jedoch die Ergebnisse etwas genauer an, kann die Operation für die richtigen Patienten zur richtigen Zeit die geeignete Methode sein.

\section{Kraniotomie bei oberflächlichen Blutungen}

Professor Hartmut Vatter von der neurochirurgischen Klinik der Universität Bonn verwies auf Subgruppenanalysen von STICH sowie auf eine 2012 veröffentlichte Metaanalyse. Nach diesen Daten zeigt eine Kraniotomie bei hämorrhagischem Schlaganfall dann einen signifikanten Nutzen, wenn die Blutung lobulär und sehr nahe an der Hirnoberfläche auftritt, wenn das Hämatomvolumen im Bereich von 20-50 ml, eventuell auch noch darüber liegt und wenn innerhalb von acht Stunden nach Blutungsbeginn operiert wird und keine intraventrikuläre Blutung zu erkennen ist. Zudem sollte der Wert auf der GCS („Glasgow Coma Scale“) im Bereich von 9 bis 12, maximal bei 15 Punkten liegen, sagte Vatter. In solchen Fällen plädiert der Neurochirurg für eine minimalinvasive Kraniotomie, bei der ein Loch in der Größe eines Zwei-Euro-Stücks in den Schädel gefräst wird, gefolgt von einem 0,5 bis $1 \mathrm{~cm}$ breiten Schnitt in den Kortex, über den man an die Blutung gelangt. Diese lässt sich dann meist gut abtragen, wobei der Chirurg nicht unbedingt das Ziel haben muss, das gesamte Hämatom auszuräumen. „Es geht lediglich um einen Massenreduktion“, so Vatter. Sie soll den Hirndruck senken sowie Ödemen und Sekundärschäden vorbeugen. Der Vorteil der minimalinvasiven Kraniotomie: Sie lässt sich schnell und mit wenig Aufwand in 30 bis 45 Minuten erledigen. Dagegen dauere die Prozedur mit modernen endoskopischen und stereotaktischen Verfahren oft zwei bis drei Stunden, weil eine lange und geräteintensive Vorbereitungszeit nötig sei.

Ein weiterer Nachteil endoskopischer Verfahren ist für Vatter, dass man das Hämatom mitunter mehrfach anstechen muss. „Das frische Hämatom ist so viskos wie Himbeergelee“, erläuterte der Chirurg, da könne man das Blut nicht einfach so absaugen.

\section{$80 \%$ überleben}

Dr. Berk Orakcioglu, neurochirurgische Universitätsklinik Heidelberg, gab Vatter zwar recht, was die oberflächennah- en Hämatome betrifft. Er hält aber bei tiefer liegenden Blutungen, wie sie etwa nach hypertensiven Entgleisungen in den Stammganglien auftreten, modernere endoskopische Verfahren für die Methode der Wahl. Dabei wird über ein kleines Bohrloch im Schädel ein Endoskop eingeführt, dessen Spitze permanent navigiert wird. Um die Blutung zu erreichen, wählen die Neurochirurgen nicht immer den kürzesten Weg, wichtig ist vielmehr, dass keine Sprachareale oder andere essenzielle Gebiete beschädigt werden. Ziel sei zudem nur das Hämatomvolumen deutlich unter $30 \mathrm{ml} \mathrm{zu}$ senken, ohne aber jedem einzelnen Erythrozyten nachzujagen.

Der Neurochirurg kann bereits auf eine erfolgreiche Serie von endoskopischen Operationen zurückblicken. Er präsentierte Daten zu 28 Patienten mit einem durchschnittlichen präoperativen Hämatomvolumen von $85 \mathrm{ml}$ - bei über $60 \mathrm{ml}$ bestehen praktisch kaum Überlebenschancen. Durch den Eingriff ließ sich das Volumen im Schnitt auf $16 \mathrm{ml}$ senken und 23 Patienten (82\%) überlebten - allerdings zum Teil mit deutlichen Behinderungen. Im Median lag der Wert auf der mRS („modifield Rankin Scale“) bei 4 Punkten, was einer moderaten bis schweren Behinderung entspricht. Aber immerhin ein Viertel der Patienten überstand die Blutung mit nur leichten Einschränkungen (mRS 1-2). Da die Methode minimalinvasiv ist, spricht nach Orakcioglus Auffassung nichts dagegen, sie auch bei Patienten unter Antikoagulanzien anzuwenden.

Weil aber viele Patienten den Eingriff nur mit schweren Behinderungen überleben, stellt sich doch die Frage, für wen die Operation überhaupt geeignet ist. „Es geht nicht darum einen komorbiden 70- oder 80-Jährigen in den Pflegezustand zu überführen", sagte der Chirurg. Auf der anderen Seite müsse man bei einem 60-Jährigen, der eine großvolumige Blutung hat und noch bei Bewusstsein ist, nicht auf eine sekundäre Verschlechterung des Zustandes warten, bevor man das Skalpell ansetze, in solchen Fällen könne man auch sofort operieren.

Thomas Müller

Kontroversen in der Intensivmedizin bei Schlaganfallpatienten, ANIM, Mannhein, 24.1.2013 